\title{
Nitration of Wheat Amylase Trypsin Inhibitors Increases Their Innate and Adaptive Immunostimulatory Potential in vitro
}

\section{OPEN ACCESS}

Edited by:

Zorica D. Juranic,

Institute of Oncology and Radiology of

Serbia, Serbia

Reviewed by:

Julio Villena,

CONICET Centro de Referencia para

Lactobacilos (CERELA), Argentina

Oscar J. Cordero,

University of Santiago de Compostela,

Spain

Ivan Milos Stankovic,

University of Belgrade, Serbia

*Correspondence:

Kurt Lucas

k.lucas@mpic.de

tThese authors have contributed equally to this work and share senior authorship

Specialty section: This article was submitted to Nutritional Immunology, a section of the journal

Frontiers in Immunology

Received: 25 July 2018 Accepted: 24 December 2018 Published: 21 January 2019

Citation:

Ziegler K, Neumann J, Liu F, Fröhlich-Nowoisky J, Cremer C

Saloga J, Reinmuth-Selzle K,

Pöschl U, Schuppan D, Bellinghausen I and Lucas K (2019) Nitration of Wheat Amylase Trypsin Inhibitors Increases Their Innate and Adaptive Immunostimulatory Potential in vitro. Front. Immunol. 9:3174. doi: 10.3389/fimmu.2018.03174
Kira Ziegler ${ }^{1}$, Jan Neumann ${ }^{1,2}$, Fobang Liu ${ }^{1}$, Janine Fröhlich-Nowoisky ${ }^{1}$, Christoph Cremer ${ }^{1,2}$, Joachim Saloga ${ }^{3}$, Kathrin Reinmuth-Selzle ${ }^{1}$, Ulrich Pöschl ${ }^{1}$, Detlef Schuppan $^{4}$, Iris Bellinghausen ${ }^{3+}$ and Kurt Lucas ${ }^{1 * t}$

${ }^{1}$ Multiphase Chemistry Department, Max Planck Institute for Chemistry, Mainz, Germany, ${ }^{2}$ Institute of Molecular Biology, Mainz, Germany, ${ }^{3}$ Department of Dermatology, University Medical Center of the Johannes Gutenberg University, Mainz, Germany, ${ }^{4}$ Institute of Translational Immunology, University Medical Center of the Johannes Gutenberg University, Mainz, Germany

Amylase trypsin inhibitors (ATI) can be found in all gluten containing cereals and are, therefore, ingredient of basic foods like bread or pasta. In the gut ATI can mediate innate immunity via activation of the Toll-like receptor 4 (TLR4) on immune cells residing in the lamina propria, promoting intestinal, as well as extra-intestinal, inflammation. Inflammatory conditions can induce formation of peroxynitrite $\left(\mathrm{ONOO}^{-}\right)$and, thereby, endogenous protein nitration in the body. Moreover, air pollutants like ozone $\left(\mathrm{O}_{3}\right)$ and nitrogen dioxide $\left(\mathrm{NO}_{2}\right)$ can cause exogenous protein nitration in the environment. Both reaction pathways may lead to the nitration of ATI. To investigate if and how nitration modulates the immunostimulatory properties of ATI, they were chemically modified by three different methods simulating endogenous and exogenous protein nitration and tested in vitro. Here we show that ATI nitration was achieved by all three methods and lead to increased immune reactions. We found that ATI nitrated by tetranitromethane (TNM) or $\mathrm{ONOO}^{-}$lead to a significantly enhanced TLR4 activation. Furthermore, in human primary immune cells, TNM nitrated ATI induced a significantly higher T cell proliferation and release of Th1 and Th2 cytokines compared to unmodified ATI. Our findings implicate a causative chain between nitration, enhanced TLR4 stimulation, and adaptive immune responses, providing major implications for public health, as nitrated ATI may strongly promote inhalative wheat allergies (baker's asthma), non-celiac wheat sensitivity (NCWS), other allergies, and autoimmune diseases. This underlines the importance of future work analyzing the relationship between endo- and exogenous protein nitration, and the rise in incidence of ATI-related and other food hypersensitivities.

Keywords: allergy, amylase trypsin inhibitors, dendritic cells, non-celiac wheat sensitivity, protein nitration, wheat

\section{INTRODUCTION}

Nitration of proteins can occur endogenously in the human body or exogenously in the environment. Air pollutants, like ozone and nitrogen oxides, are able to nitrate allergenic proteins, like the major birch pollen allergen Bet $\mathrm{v} 1$, leading to an increased allergic potential and elevated immune reactions (1-6). In the human body, inflammatory conditions can lead 


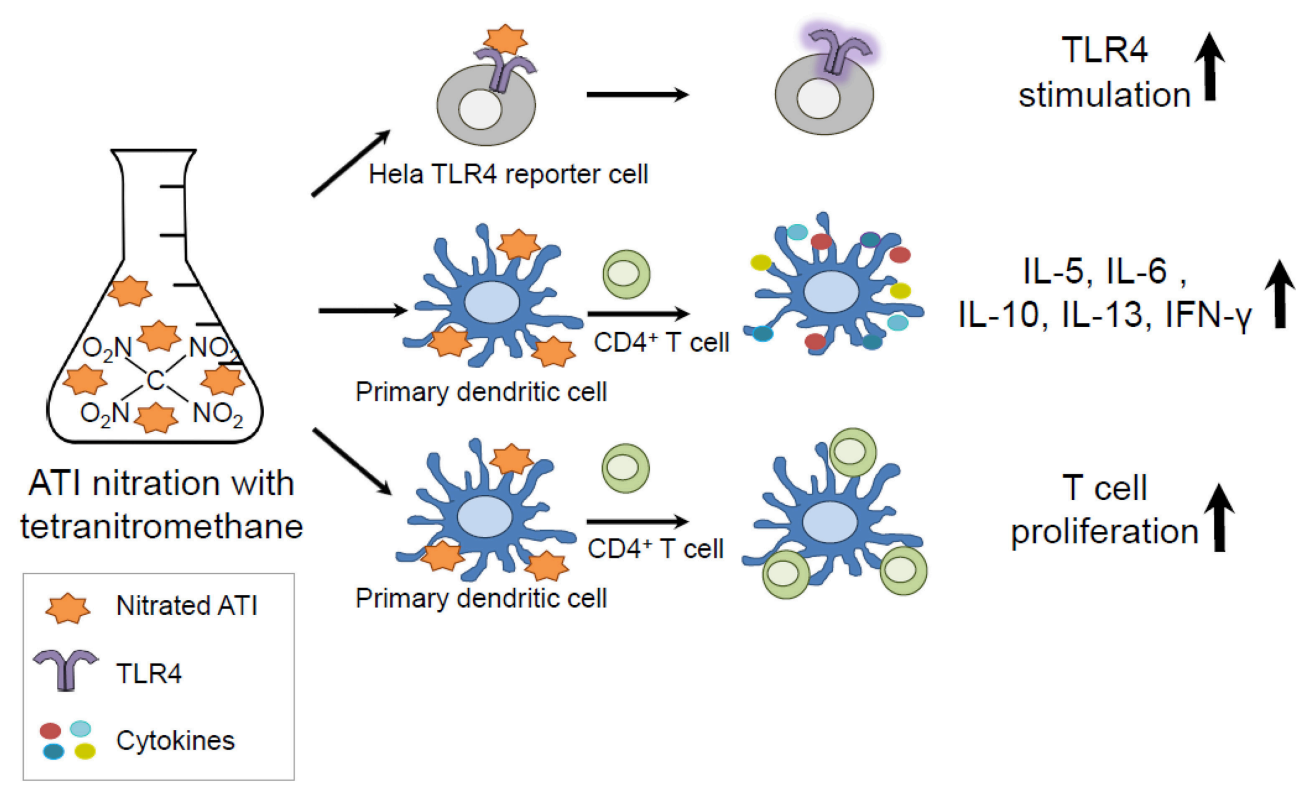

GRAPHICAL ABSTRACT | Amylase Trypsin Inhibitors (ATI) can be found in all gluten containing grains and are capable to mediate innate immunity by direct TLR4 stimulation. As protein nitration can occur during inflammatory processes in the body and under environmental conditions, ATI were nitrated and tested in vitro. The nitrated ATI induced increased innate and adaptive immune reactions.

to the formation of $\mathrm{ONOO}^{-}$, which is the main agent for endogenous protein nitration (5, 7-9). Moreover, several air pollutants and pathogens can induce or favor inflammatory processes and related nitration processes $(1,3,6)$. Diseases typically accompanied by a high degree of inflammation are, for example, autoimmune and other chronic inflammatory diseases, like chronic obstructive pulmonary disease (COPD), allergic asthma, inflammatory bowel diseases (IBD), or non-celiac nonallergy wheat sensitivity (NCWS) (10-12).

We previously showed that amylase trypsin inhibitors (ATI), a family of non-gluten proteins, are responsible for manifestations of mainly extra-intestinal symptoms of NCWS (10, 11, 13, 14). Moreover, ATI are major allergens in baker's asthma, a classical IgE mediated allergy $(15,16)$. ATI can be found in all gluten containing grains (wheat, barley, rye), and represent $2-4 \%$ of the total protein (17). In plants, ATI regulate the germination processes (18) and defense mechanisms by blocking the amylase and trypsin activity of parasites (19). The wheat intake of an adult person is about $250 \mathrm{~g}$ per day, mainly as processed bread or pasta which is equivalent to $0.5-1 \mathrm{~g}$ ATI (10). Remarkably, ATI are resistant to food processing and to proteolysis in the gastrointestinal tract where they stay biologically active (20). In the gut ATI are able to stimulate immune cells residing in the lamina propria and mesenteric lymph nodes through TLR4 binding and stimulation, and the emigration of the activated myeloid cells (20-22).

The innate immune receptor TLR4 recognizes damage and pathogen associated patterns (DAMPs/PAMPs), like lipopolysaccharides (LPS), which are major components in the outer membrane of gram negative bacteria. Upon stimulation, the receptor triggers an NF- $\kappa \mathrm{B}$ dependent cascade leading to the release of pro-inflammatory cytokines $(23,24)$. Importantly, ATI can trigger TLR4 by direct interaction, and provoke innate immunity $(21,22)$. In a mouse model of inflammatory bowel disease, ATI enhanced the dextran sodium sulfate-induced intestinal inflammation by increasing the number of activated macrophages and dendritic cells in all sections of the intestine, the lamina propria, and especially in the mesenteric lymph nodes (20). Moreover, we showed in two mouse studies on experimental airway inflammation that ATI-enriched diets not only enhanced allergen-induced intestinal, but also lung allergic responses in an IgE- and TLR4-dependent manner $(25,26)$. Thus, the adjuvant effect of ATI is not limited to the intestine, but can also be observed for other organs, fueling ongoing inflammation. These inflammatory conditions might favor the formation of $\mathrm{ONOO}^{-}$and, thereby, protein nitration $(7-9,27)$, including ATI in the gut, apart from the environmental factors that may induce protein nitration.

To elucidate the effect of nitration, we chemically modified ATI using three different methods simulating endo- (in vivo) and exogenous (environmental) nitration mechanisms. These nitrating agents mainly induce the formation of 3 nitrotyrosine $(1,5,27-31)$. The aim of our study was to examine whether nitrated ATI exhibit an altered innate and adaptive immunostimulatory capacity. Modified ATI were quantified for their nitration degree using HPLC-DAD and ELISA. Furthermore, various in vitro studies were performed. A novel HeLa TLR4 reporter cell line was established for determination of innate immune stimulation. Adaptive immune reactions were analyzed in a mixed lymphocyte reaction by the use of primary immune cells isolated from whole blood of healthy donors. TLR4 
activation, NF- $\kappa$ B translocation, expression of surface maturation markers, Th1, Th2, relevant cytokines, and T cell proliferation were measured.

\section{MATERIALS AND METHODS}

\section{Nitration and Analysis of Nitrated ATI Nitration With Tetranitromethane (TNM) of ATI}

ATI were obtained from Sigma Aldrich ( $\alpha$-Amylase Inhibitor from Triticum aestivum (wheat seed), Sigma-Aldrich, Darmstadt, Germany). Aliquots of aqueous ATI solutions ( $1 \mathrm{mg} / \mathrm{mL}, 0.5 \mathrm{~mL}$ ) were mixed with $4.55 \mu \mathrm{L}$ TNM/Methanol (4\%,v/v) and stirred for $3 \mathrm{~h}$ at room temperature. To remove excess TNM after the reaction, a $\mathrm{PD}-25$ size exclusion chromatography column (GE Healthcare, Little Chalfont, Great Britain) was used according to the manufacturer's instructions. Finally, the ATI were eluted with endotoxin free water (MilliQ, Biopak, Merck, Darmstadt, Germany).

\section{Nitration With $\mathrm{O}_{3} / \mathrm{NO}_{2}$ of ATI}

ATI aqueous solutions $(0.5 \mathrm{mg} / \mathrm{mL}, 1 \mathrm{~mL})$ were exposed to a gas mixture of $\mathrm{O}_{3}$ and $\mathrm{NO}_{2}$, as described previously (31). Briefly, $\mathrm{O}_{3}$ was produced from synthetic air passed through a UV lamp (LOT-Quantum Design, Darmstadt, Germany) at $\sim 1.98 \mathrm{~L} / \mathrm{min}$. The air flow was then mixed with a $\mathrm{N}_{2}$ flow $(20 \mathrm{~mL} / \mathrm{min})$ containing $\sim 5 \mathrm{ppmV} \mathrm{NO}$ (AirLiquide, Düsseldorf, Germany). The resulting air gas mixtures were bubbled directly through the aqueous ATI solutions at a flow rate of $60 \mathrm{~mL} / \mathrm{min}$ using a Teflon tube (ID: $1.59 \mathrm{~mm}$ ). The concentrations of $\mathrm{O}_{3} / \mathrm{NO}_{2}$ were monitored by commercial monitoring instruments (Ozone analyzer, 49i; $\mathrm{NO}_{\mathrm{x}}$ analyzer, 42i-TL, Thermo Fisher Scientific, Darmstadt, Germany, respectively).

\section{ONOO- Nitration of ATI}

ATI solutions were prepared in $50 \mathrm{mM}$ ammonium bicarbonate buffer at pH 7.8 (Carl Roth, Karlsruhe, Germany). For one reaction $300 \mu \mathrm{L}$ of ATI solution [ $1 \mathrm{mg} / \mathrm{mL}$ ] were mixed with 2.85 $\mu \mathrm{L} \mathrm{ONOO}^{-}$(160-200 mM, Merck) in brown reaction vessels (Eppendorf, Hamburg, Germany) and incubated for $110 \mathrm{~min}$ on ice. Immediately after the reaction, the samples were desalted using a PD-10 size exclusion mini column (GE Healthcare), following the manufacturer manual with endotoxin free water.

\section{HPLC-DAD Analysis}

All nitrated ATI samples were analyzed using HPLC coupled to diode array detection (HPLC-DAD, 1,200 series, Agilent Technologies, Santa Clara, California, USA). Values were determined using peak areas of signals at wavelengths 280 and $357 \mathrm{~nm}$. A detailed description of the analytical method can be found in Selzle et al. (30). The nitration degree is defined as the concentration of nitrotyrosine as a fraction of the sum of the concentrations of nitrotyrosine and tyrosine. For example, ATI 0.19 Chain D from T. aestivum comprises five tyrosine residues. A nitration degree of $20 \%$ reflects on average one nitrotyrosine per ATI 0.19 molecule.

\section{Endotoxin Quantification}

Endotoxin was quantified by Limulus Amebocyte Lysate chromogenic endotoxin quantitation kit (Thermo Fisher Scientific). ATI samples were tested at several dilutions and compared to an Escherichia coli endotoxin standard (011:B4) provided with the kit. The endotoxin levels in the final concentration used for all experiments were $<20$ Endotoxin units per $\mathrm{mL}$.

\section{Protein Analysis}

To determine the ATI protein concentrations before and after nitration, a bicinchoninic acid assay (Thermo Fisher Scientific) was used according to the manufacturer's instructions. The optical density at $562 \mathrm{~nm}$ was determined using a Synergy Neo plate reader (Biotek, Bad Friedrichshall, Germany).

ATI oligomers were detected using sodium dodecyl sulfate polyacrylamide gel electrophoresis (5-20\%, Bio-Rad, Munich, Germany), referring to the instruction manual. A $5 \mu \mathrm{g}$ portion of each sample was prepared in 2x Laemmli buffer (Bio-Rad) containing $100 \mathrm{mM}$ Dithiothreitol (Sigma Aldrich), heated on $96^{\circ} \mathrm{C}$ for $5 \mathrm{~min}$, and loaded on the gel. After separation, the gel was stained for $3 \mathrm{~h}$ in Coomassie blue (Bio-Rad) and unstained in an aqueous solution containing 10\% methanol (Merck) and 20\% acetic acid (Carl Roth) over night. For image acquisition and for analysis, a ChemiDoc system and Image Lab software 5.2.1 (both Bio-Rad) were used, respectively.

\section{Cell Culture}

\section{Hela TLR4 Dual Luciferase Reporter Cell Line (HeLa TLR4 Dual)}

Cells were grown in Dulbecco's Modified Eagle's Medium (DMEM, Thermo Fisher Scientific) containing $25 \mathrm{mM} \mathrm{D-}$ glucose, $1 \mathrm{mM}$ sodium pyruvate supplemented with $10 \%$ heatinactivated fetal calf serum (FCS), (Biochrom, Berlin, Germany), 1\% Penicillin/Streptomycin (Thermo Fisher Scientific), and $140 \mu \mathrm{g} / \mathrm{mL}$ Hygromycin B (Invivogen, Toulouse, France) in a humidified atmosphere of $5 \% \mathrm{CO}_{2}$ at $37^{\circ} \mathrm{C}$.

For simultaneous determination of TLR4 stimulation and viability, a novel monoclonal dual reporter cell line was established. Therefore, the HeLa TLR4 cell line (Novusbio, Wiesbaden, Germany), expressing Renilla luciferase under the control of an IL-8 promotor reporting TLR4 activity, was stably transfected with a plasmid, constitutively expressing Firefly luciferase, and, thereby, measuring viability (pCMB-firefly-luchygro, kindly provided by Ernesto Bockamp, University Medical Center of the Johannes Gutenberg University). Lipofectamine 3000 (Thermo Fisher Scientific) was used as the transfecting reagent according to the manufacturer's protocol.

\section{Combined TLR4 and viability assay}

20,000 HeLa TLR4 dual reporter cells were seeded in a flat bottom 96-well plate (Greiner, Frickenhausen, Germany) in 100 $\mu \mathrm{L}$ complete DMEM. On the next day, the cells were treated with different nitrated ATI at a final concentration of $7.5 \mu \mathrm{g} / \mathrm{mL}$. Mock nitrations and medium served as negative controls, and LPS EB ( $25 \mathrm{ng} / \mathrm{mL}$, Invivogen) as a positive control. After $7 \mathrm{~h}$, the plate was washed with $200 \mu \mathrm{L}$ of warm PBS containing 
calcium and magnesium (Thermo Fisher Scientific). Then the cells were lysed by adding passive lysis buffer (Dual-luciferase reporter assay, Promega, Mannheim, Germany) and frozen at $-80^{\circ} \mathrm{C}$. An analysis of both luciferase reporter activities in the cell lysate was performed according to the manufacturer's manual (Promega). The relative luciferase activity was calculated by dividing the Renilla luciferase (TLR4) signal by the Firefly luciferase (viability) signal. The resulting values were normalized to the value obtained for LPS treated cells.

To inhibit TLR4 signaling, HeLa TLR4 dual cells were pre-incubated with the TLR4 antagonist TAK242 $(0.36 \mu \mathrm{g} / \mathrm{mL}$, Merck), or, as a negative control, its solvent dimethylsulfoxide $(4.4 \mu \mathrm{g} / \mathrm{mL}$, Thermo Fisher Scientific) for $150 \mathrm{~min}$.To provide a stronger stimulation, the doses of ATI were doubled in these experiments $[15 \mu \mathrm{g} / \mathrm{mL}]$.

\section{Generation of Monocyte Derived Dendritic Cells (DC)}

Buffy coats from ten healthy donors were obtained from the Transfusion Center (University Medical Center of the Johannes Gutenberg University, Mainz, Germany) with approval from the local ethical committee (Landesärztekammer Rheinland-Pfalz). Peripheral blood mononuclear cells (PBMC) were isolated by Ficoll-Paque $1.077 \mathrm{~g} / \mathrm{mL}$ (Biochrom) density centrifugation. The autologous plasma was heat-inactivated at $56^{\circ} \mathrm{C}$ for $30 \mathrm{~min}$, centrifuged at $1,500 \times \mathrm{g}$, and stored at $4^{\circ} \mathrm{C}$. To enrich $\mathrm{CD} 14^{+}$ monocytes, $5 \times 10^{6}$ PBMC per well were incubated in a 12 -well plate (Greiner) in $1.5 \mathrm{~mL}$ Iscove modified Dulbecco medium (IMDM, Lonza, Basel, Switzerland) enriched with $1 \%$ antibiotic/antimycotic solution (Sigma Aldrich) and 3\% autologous plasma for $40 \mathrm{~min}$ in a cell incubator under $5 \% \quad \mathrm{CO}_{2}$ atmosphere at $37^{\circ} \mathrm{C}$. Cells were washed 3 times with warm PBS (without calcium magnesium, Thermo Fisher Scientific), and maintained in IMDM, supplemented with 10 ng/mL IL-4 (Miltenyi, Bergisch Gladbach, Germany), 200
$\mathrm{U} / \mathrm{mL}$ granulocyte-macrophage colony-stimulating factor (GMCSF, Leukine ${ }^{\circledR}$, Immunex Corp., Seattle, WA, USA), and $2 \%$ autologous plasma. On day 6, immature DC were pulsed with ATI $[15 \mu \mathrm{g} / \mathrm{mL}]$, TNM nitrated ATI $[15 \mu \mathrm{g} / \mathrm{mL}]$, equivalent amounts of mock nitrated solution, or were left untreated. To induce DC maturation, the cells were additionally treated with tumor necrosis factor (TNF)-alpha, $10 \mathrm{ng} / \mathrm{mL}$, Miltenyi Biotec), IL-1 $\beta$ (10 ng/mL, Miltenyi Biotec) and prostaglandin $\mathrm{E}_{2}$ $(1 \mu \mathrm{g} / \mathrm{mL}$, Cayman Chemical, Ann Arbor, MI, USA). After $48 \mathrm{~h}$, DC were harvested, washed twice in cold PBS, and used for T cell stimulation assays as well as for analysis of surface marker expression.

\section{Surface marker staining and analysis by flow cytometry}

$5 \times 10^{4} \mathrm{DC}$ or $5 \times 10^{5} \mathrm{~T}$ cells were stained with specific mouse anti-human monoclonal antibodies (mAbs) for $20 \mathrm{~min}$ at $4{ }^{\circ} \mathrm{C}$. The following antibodies were used: AlexaFluor $647-$ conjugated CD4 (MT310; Santa Cruz Biotechnology, Inc., Santa Cruz, CA, USA), fluorescein isothiocyanate (FITC)conjugated human leukocyte antigen D-related (HLA-DR) (L243), phycoerythrin (PE)-conjugated CD80 (L307.4), and allophycocyanin-conjugated CD83 (HB15e, all from BD Biosciences). As a negative control, matured cells were used. Then, cells were washed, and analyzed by BD Accuri ${ }^{\mathrm{TM}}$ C6 Plus Flow Cytometer (BD Biosciences).

\section{Isolation of $\mathrm{CD}^{+} T$ cells and co-culture with autologous native ATI- or TNM nitrated ATI-pulsed DC}

Autologous $\mathrm{CD}^{+} \mathrm{T}$ cells were obtained from PBMC using antibody-coated paramagnetic MicroBeads (MACS; Miltenyi Biotec) according to the manufacturer's protocol. Separation was confirmed by flow cytometry (purity, $>98 \% \mathrm{CD}^{+} \mathrm{T}$ cells).

$1 \times 10^{5} \mathrm{~T}$ cells and $1 \times 10^{4} \mathrm{mDC}$ were co-cultured in 96well plates (Greiner) in triplicates in $200 \mu \mathrm{L}$ IMDM containing
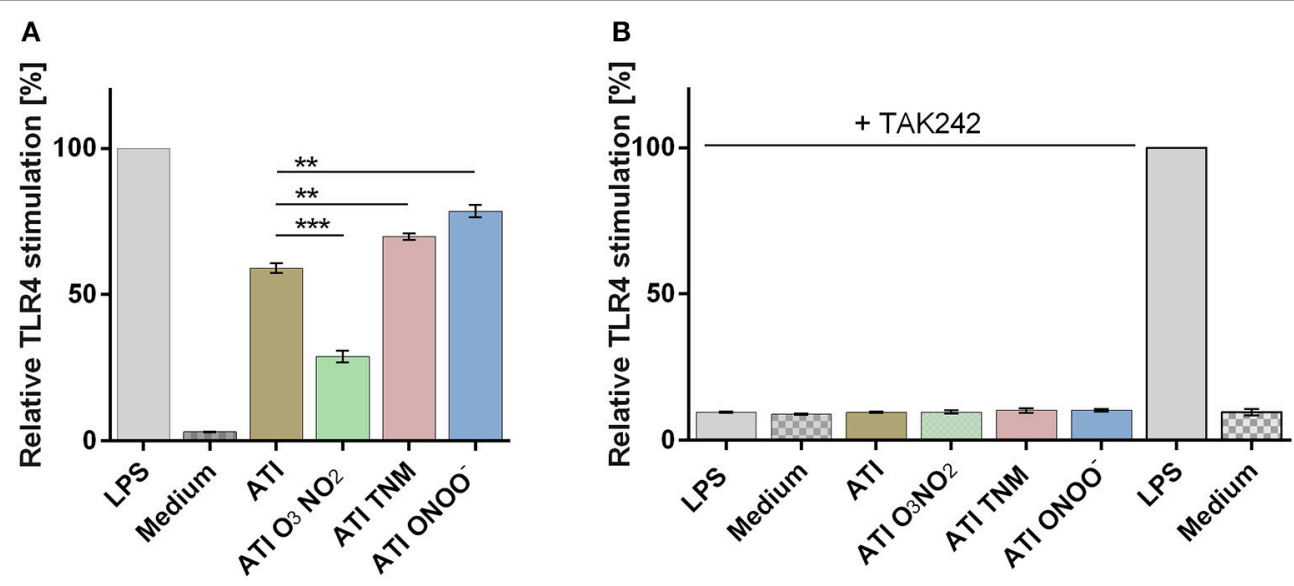

FIGURE 1 | Different nitrated ATI induce distinct TLR4 activation in HeLa TLR4 dual reporter cells. (A) HeLa TLR4 dual reporter cells were treated for $7 \mathrm{~h}$ with nitrated or unmodified ATI $[7.5 \mu \mathrm{g} / \mathrm{mL}]$ or LPS [25 ng/mL] as a positive control. (B) Cells were pre-incubated for $2.5 \mathrm{~h}$ with TAK242 [0.36 $\mu \mathrm{g} / \mathrm{mL}]$ or its solvent DMSO [4.4 $\mu \mathrm{g} / \mathrm{mL}]$. Then the cells were stimulated with nitrated or unmodified ATI [ $15 \mu \mathrm{g} / \mathrm{mL}]$, or LPS [ $25 \mathrm{ng} / \mathrm{mL}]$ for $7 \mathrm{~h}$. The relative luciferase activity was calculated by dividing the Renilla luciferase (TLR4) signal by the Firefly luciferase (viability) signal. The resulting values were normalized to the value obtained for LPS- treated cells. Shown are the means $\pm \mathrm{SD}$ of three independent experiments measured in triplicates using two independently nitrated probes. ${ }^{\star \star \star} P<0.001$, ${ }^{\star \star} P<0.01$. 
$5 \%$ autologous plasma. Five days later, $50 \mu \mathrm{L}$ of supernatant was taken for quantification of cytokine production. For the determination of proliferation, the co-culture was pulsed with 37 $\mathrm{kBq} /$ well of $\left[{ }^{3} \mathrm{H}\right]$-thymidine (ICN Biomedicals, CA, USA) for $6 \mathrm{~h}$ and $\left[{ }^{3} \mathrm{H}\right]$-TdR incorporation was measured using a beta counter (1205 Betaplate, LKB Wallac, Turku, Finland).

\section{Quantification of soluble cytokines by magnetic multiplex assay}

For quantification of cytokines, multiplex assay kits (R\&D systems, Biotechne, Wiesbaden, Germany) were used. Supernatants from immature DC cultures were tested for TNF-alpha, IL-6, IL-8, IL-1 $\beta$, and monocyte chemoattractant protein 1 (MCP1). Supernatants of mature DC-T cell co-cultures were analyzed for interferon gamma (IFN-gamma), IL-10, IL-17, IL-6, IL-4, IL-5, and IL-13. The samples were prepared according to the manufacturer's manual and analyzed on a MAGPIX device (Luminex, Austin, Texas, USA).

\section{Quantification of ATI Induced NF-kB Translocation}

Nuclear NF- $\kappa$ B translocation in macrophages was determined by fluorescence microscopy. Therefore, PBMCs were isolated and seeded in 12-well glass bottom plates (Cellvis, California, USA) at a concentration of $1.5 \times 10^{6}-2 \times 10^{6}$ cells per well in IMDM enriched with $200 \mathrm{U} / \mathrm{mL}$ GM-CSF and $2 \%$ autologous plasma. After 6 days, cells were stimulated for $2 \mathrm{~h}$ either with ATI, ATI TNM [12.5 $\mu \mathrm{g} / \mathrm{mL}$ ], or with LPS EB (100 ng/mL, Invivogen) as a positive control. Untreated cells (medium) served as a negative control. Next, cells were washed once in pre-warmed PBS and fixed using 4\% formaldehyde solution (Thermo Fisher
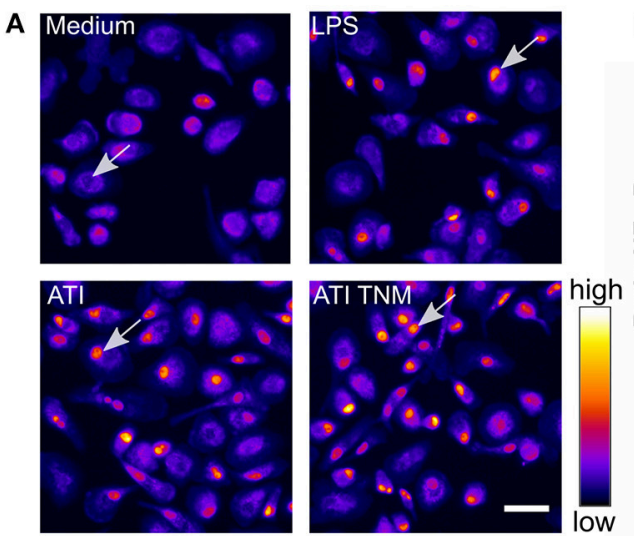

B
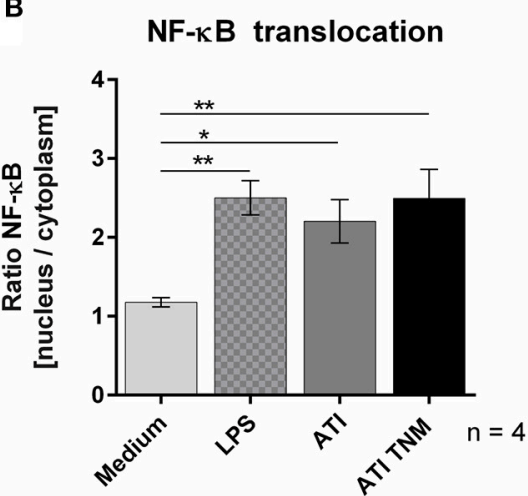

C

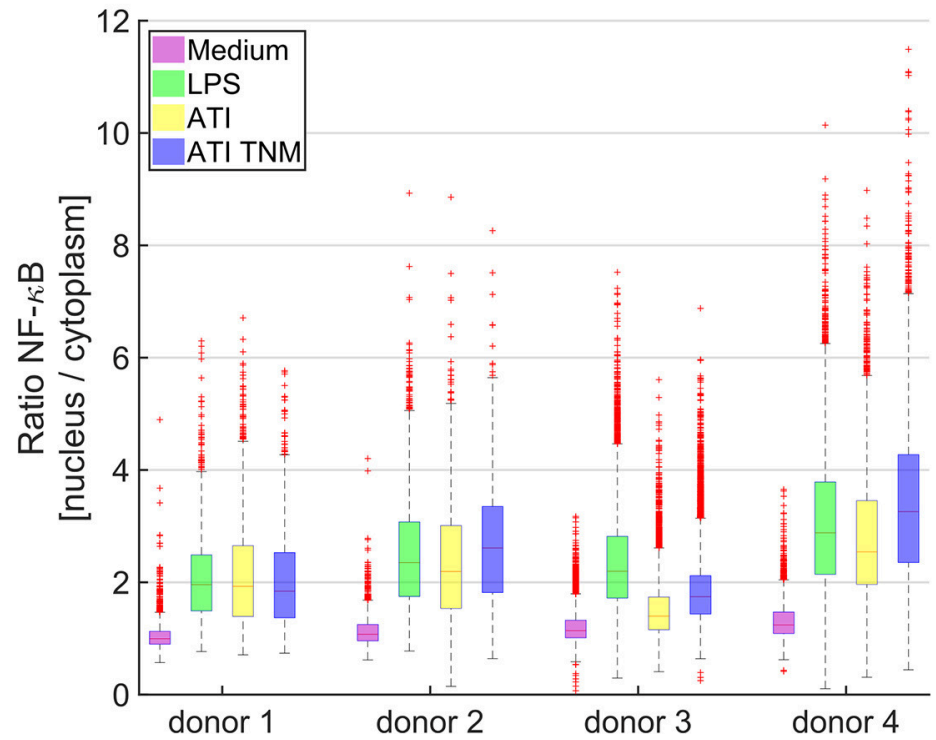

FIGURE 2 | Increased NF-kB p65 translocation into the cell nucleus in ATI and ATI TNM treated primary human macrophages. (A) Fluorescence microscopy images of macrophages treated for $2 \mathrm{~h}$ with LPS (positive control, $100 \mathrm{ng} / \mathrm{mL}$ ), ATI or ATI TNM [12.5 $\mu \mathrm{g} / \mathrm{mL}]$ and stained for NF-kB. In untreated conditions (medium) NF-kB resides mostly in the cytoplasm, whereas an increased translocation of NF-kB p65 into the nucleus can be observed for LPS, ATI, and ATI TNM treated cells (indicated by arrows). Brightness and contrast were adjusted to the same level for every image within the panel. Scale bar $=50 \mu \mathrm{m}$. (B) Quantitative evaluation of NF-kB translocation from the cell cytoplasm to the nucleus by Harmony high-content analysis software (Perkin Elmer). Shown are the means \pm SEM from four independent experiments/donors, ${ }^{*} P<0.05,{ }^{\star *} P<0.01$. (C) Donor-dependent response to LPS (green), ATI (yellow), and ATI TNM (blue) treatment. 
Scientific) in PBS for $10 \mathrm{~min}$ at $37^{\circ} \mathrm{C}$. Subsequently, cells were rinsed three times with $\mathrm{PBS}$, then blocked and permeabilized in PBS containing $0.3 \%$ Triton X-100 (Merck) and 5\% bovine serum albumin (BSA, Cell Signaling Technology, Danvers, Massachusetts) for $1 \mathrm{~h}$ at room temperature. Afterwards, cells were incubated with primary rabbit anti-NF- $\kappa \mathrm{B}$ p65 mAb (D14E12, Cell Signaling Technology) diluted in PBS comprising $1 \%$ BSA and $0.3 \%$ Triton X-100 (Merck) overnight at $4^{\circ} \mathrm{C}$. Thereafter, cells were washed three times in PBS for $5 \mathrm{~min}$ each and incubated for $1 \mathrm{~h}$ at room temperature with anti-rabbit
Alexa Fluor 568 antibody (A-11011, Thermo Fisher Scientific) diluted in PBS comprising 1\% BSA and $0.3 \%$ Triton X-100. Cells were washed again three times in PBS for 5 min each and cell nuclei were counterstained using 4',6-diamidino-2-phenylindole (DAPI, Thermo Fisher Scientific) according to the manufacturer's instructions. Imaging of stained macrophages was performed at an Opera Phenix High-Content Screening system (Perkin Elmer, Waltham, Massachusetts, USA). PBS was used as an imaging buffer. For image analysis, Harmony high-content analysis software (Perkin Elmer, Waltham, Massachusetts, USA)
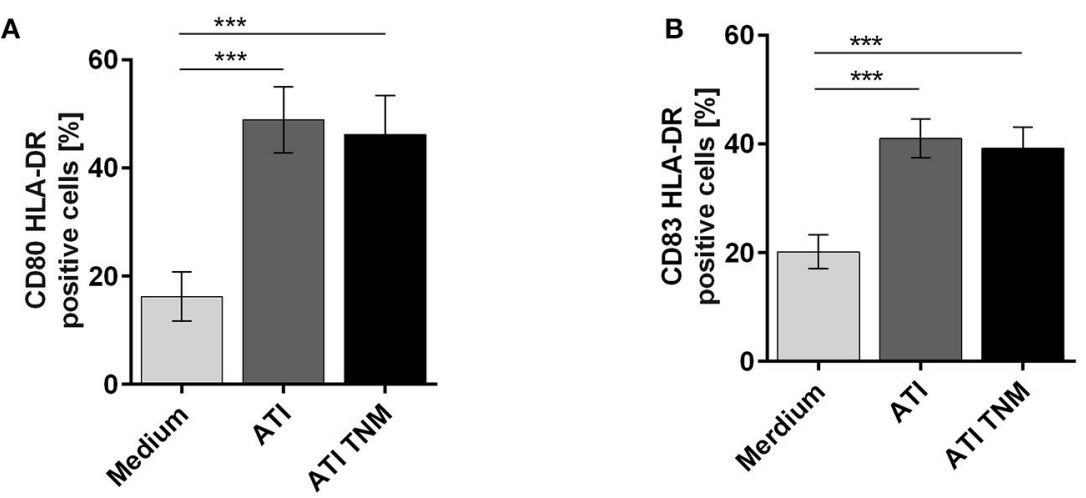

C

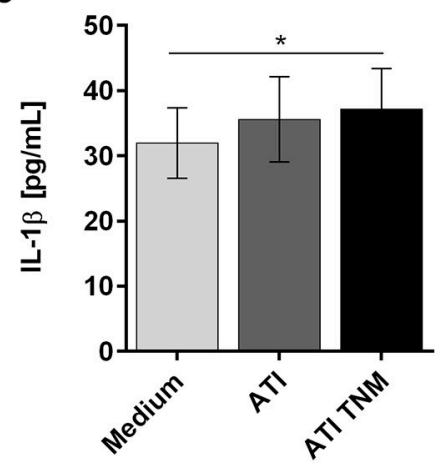

D
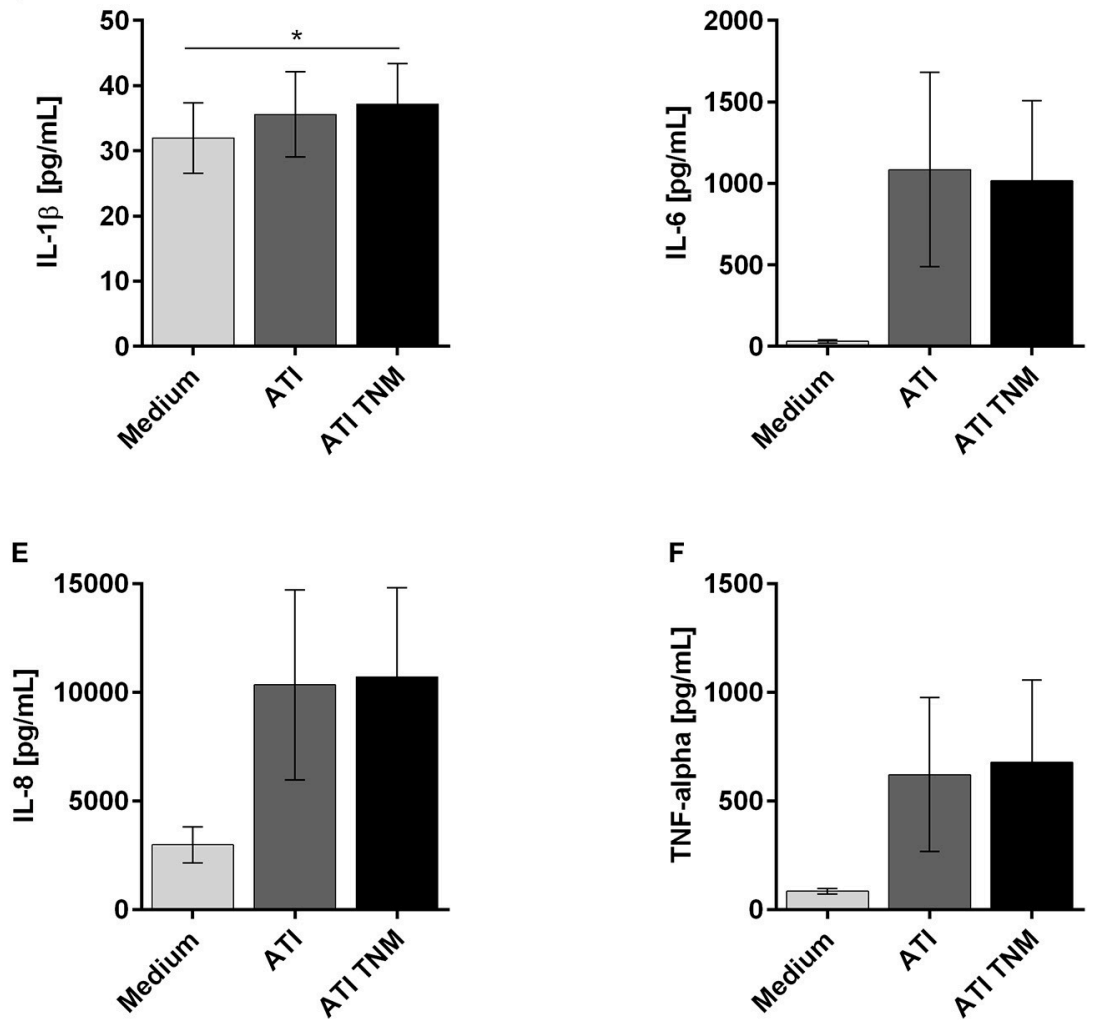

FIGURE 3 | Enhanced expression of maturation markers as well as pro-inflammatory cytokines by human immature DCs upon stimulation with ATI and ATI TNM. Immature DCs were pulsed with ATI or ATI TNM $[15 \mu \mathrm{g} / \mathrm{mL}]$ on day 6 . Forty-eight hours later cells were stained for expression of the indicated surface markers and analyzed by flow cytometry (A,B). Before, supernatants were taken for determination of IL-1 $\beta$, IL-6, IL-8, and TNF-alpha by magnetic multiplex assay (C-F). Shown are the means \pm SEM from ten independent experiments/donors, ${ }^{\star} P<0.05,{ }^{* \star *} P<0.001$ 
was used. Nuclei were identified by DAPI staining, and a ring-like mask around each identified nucleus was created to obtain the corresponding cytoplasm area. Afterwards, the mean intensities of NF- $\kappa \mathrm{B}$ within both masks were determined and the nuclear to cytoplasmic ratio of NF- $\mathrm{B}$ was calculated. The described image processing steps are illustrated in Figure S1.

\section{Statistics}

GraphPad Prism version 6.07 (GraphPad, San Diego, California) was used for statistical analysis. To determine the standard deviation between the different treated groups, an ANOVA was performed. Unpaired $t$-tests were performed for TLR4 activation measurements using the Hela TLR4 dual reporter cell line. For all data sets based on primary immune cells, paired $t$-tests were carried out. Differences between groups were considered as significant when ${ }^{*} p<0.05,{ }^{* *} p<0.01$, or ${ }^{* * *} p<0.001$. The number of experiments performed for each data set is described at the end of each figure legend.

\section{RESULTS}

\section{Nitration of ATI by Different Nitrating Agents}

The degree of ATI nitration varied for the different nitrating agents and methods used. High nitration was observed with TNM and $\mathrm{ONOO}^{-}$. Lowest nitration was obtained for $\mathrm{O}_{3} / \mathrm{NO}_{2}$ (Table 1). All three nitration methods were able to modify ATI and induce about $3-13 \%$ of covalently linked ATI oligomers (Figures S2A,B).

\section{Nitration of ATI Enhances TLR4 Stimulatory Activity}

Compared to unmodified ATI, TNM, and $\mathrm{ONOO}^{-}$, nitrated ATI induced a significantly higher TLR4 activation in HeLa TLR4 dual reporter cells. However, ATI nitrated with $\mathrm{O}_{3}$ and $\mathrm{NO}_{2}$ exhibited a lower TLR4 activation compared to native ATI (Figure 1A). All mock nitrations showed no TLR4 activation (Figure S3).

Administration of the TLR4 antagonist TAK242 completely diminished TLR4 activation induced by ATI or by the positive control LPS (Figure 1B).

TABLE 1 | Levels of ATI nitration by different nitrating agents.

\begin{tabular}{llll}
\hline Agent & Reaction condition & $\begin{array}{l}\text { Nitration } \\
\text { degree (\%) }\end{array}$ & $\begin{array}{l}\text { Nitrotyrosine } \\
{[\mu \mathbf{g} / \mathbf{m l}]^{\mathbf{b}}}\end{array}$ \\
\hline $\mathrm{TNM}$ & $4.55 \mu \mathrm{L}[4 \%], 3 \mathrm{~h}$ & $19.5 \pm 0.2$ & $0.36 \pm 0.03$ \\
$\mathrm{O}_{3} / \mathrm{NO}_{2}$ & $\begin{array}{l}\mathrm{O}_{3}[200 \mathrm{ppb}], \mathrm{NO}_{2} \\
{[50 \mathrm{ppb}], 4 \mathrm{~h}}\end{array}$ & $8.6 \pm 0.5$ & $7.8 \mathrm{E}-04 \pm 2.4 \mathrm{E}-04$ \\
$\mathrm{ONOO}^{-}$ & $2.85 \mu \mathrm{l}[200 \mathrm{mM}], 1.8 \mathrm{~h}$ & $26.2 \pm 6,2$ & $0.23 \pm 0.12$
\end{tabular}

a The ND values here were corrected according to the background for pre-nitrated signals of native ATI $(n=2)$. Blank samples were conducted under the same nitration conditions, and no signal at $280 \mathrm{~nm}$ and $357 \mathrm{~nm}$ was detected for all the mock samples.

${ }^{b}$ The values are expressed in equivalents of nitrotyrosines $[\mu \mathrm{g}]$ nitrated BSA nBSA per $1 \mu \mathrm{g}$ of nitrated ATI solution $(n=2)$. The values were corrected against unmodified ATI solution. Mock samples showed no nitration.

\section{Nitrated and Unmodified ATI Induce Similar Activation of Macrophages and Immature DC}

In unstimulated primary human macrophages, NF- $\kappa \mathrm{B}$ is mostly located in the cytoplasm. Upon treatment with ATI or TNM nitrated ATI, a significantly enhanced NF- $\mathrm{KB}$ translocation from cytoplasm to the cell nucleus was observed using fluorescence microscopy (Figure 2A). Nitrated ATI exhibited only slightly higher NF- $\kappa B$ translocation compared to native ATI, which was also observed in the individual donor dependent responses (Figures 2B,C).

Immature DC treated with unmodified or TNM nitrated ATI expressed significantly higher amounts of CD80, CD83, and HLA-DR on their surface in comparison to untreated cells (Figures 3A,B). For DC treated with mock nitrated samples, no changes were observed (Figures S4A,B). Furthermore, the release of the pro-inflammatory cytokines IL-1 $\beta$, IL-6, IL-8, and TNF-alpha by immature DC was similarly elevated upon ATI or ATI TNM treatment compared to untreated cells (Figures 3C-F). No significant changes were observed for MCP-1 release or cells treated with mock nitrations (Figures S4C-G).

\section{Nitrated ATI Enhance Proliferation and Induce an Enhanced Th1 and Th2 Cytokine Expression Profile in CD4 ${ }^{+} \mathrm{T}$ Cells Stimulated With Autologous Mature DC}

To analyze the immunogenicity of nitrated vs. unmodified ATI, $\mathrm{CD}^{+} \mathrm{T}$ cells were co-cultured with autologous mature DC treated before with ATI or ATI-TNM. Proliferations as well as production of Th1 and Th2 cytokines were analyzed. In general, $\mathrm{CD}^{+} \mathrm{T}$ cells from almost all donors showed an enhanced proliferative response and cytokine release after stimulation with ATI-pulsed compared to untreated DC. Only DC treated with nitrated ATI induced a significantly increased $\mathrm{T}$ cell proliferation (Figure 4A). Moreover, the release of the Th2 cytokines IL5, IL-6, IL-10, and IL-13 as well as the Th1 cytokine IFNgamma was significantly enhanced after stimulation with DC treated with ATI-TNM (Figures 4B-F). Comparing both ATItreated groups, we found a significant higher release for IL5, IL-6, IL-10, and IFN-gamma upon the ATI-TNM stimulus (Figures 4B,D,F). There were no significant changes in IL-4 and IL-17A release between all groups (Figures S5A,B). Mock nitration did neither influence cytokine release nor $\mathrm{T}$ cell proliferation (Figures S5C-H).

\section{DISCUSSION}

In the present study, we analyzed the impact of nitration on the immunogenicity of ATI, and could show that ATI can be nitrated by exogenous and endogenous nitrating agents. Furthermore, we demonstrated that nitration of ATI lead to enhanced innate and adaptive immune responses compared to unmodified ATI (Graphical Abstract).

By using a novel HeLa TLR4 dual luciferase reporter cell line, we could demonstrate that TNM and $\mathrm{ONOO}^{-}$mediated ATI nitration induced significant enhanced TLR4 stimulation, 

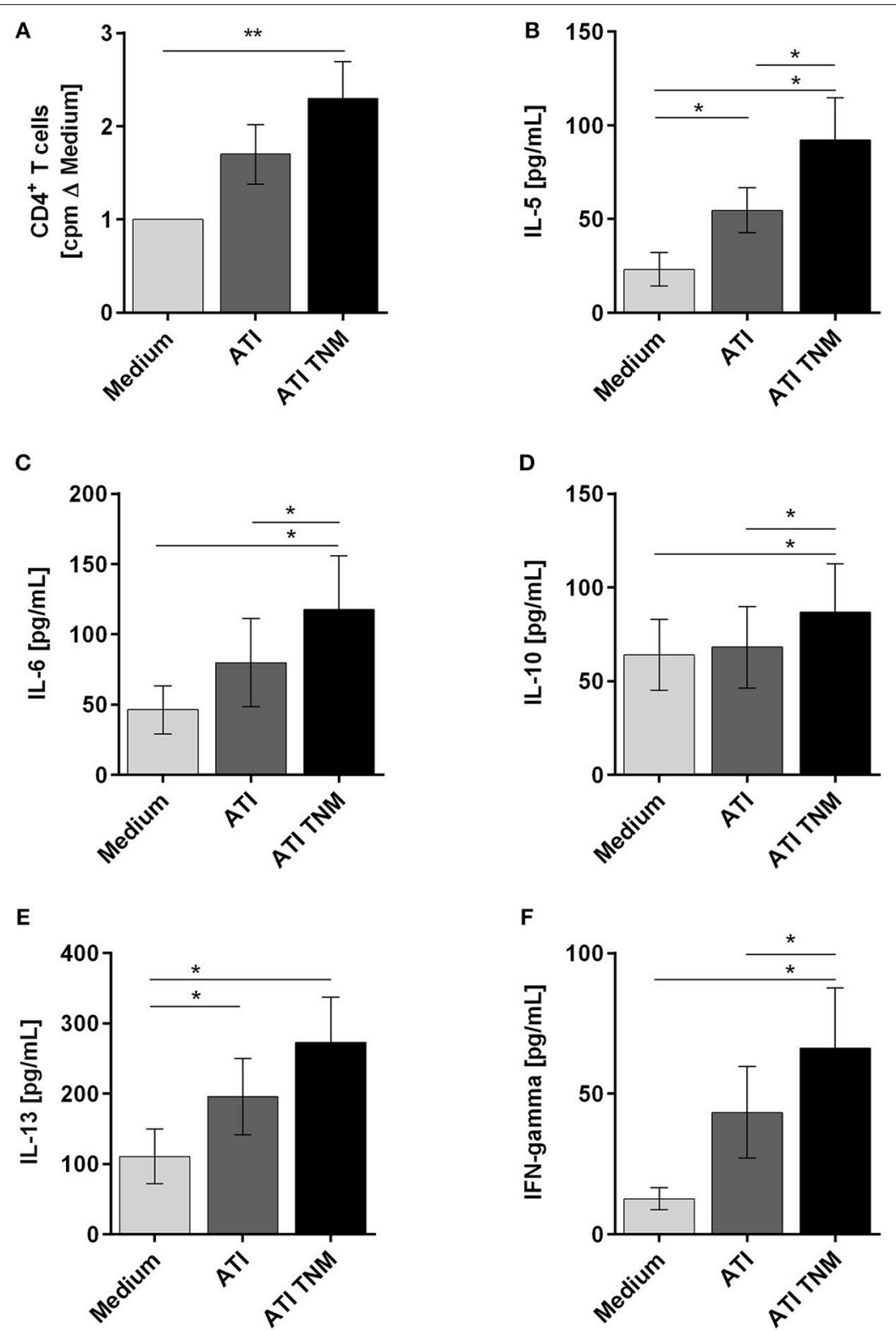

FIGURE 4 | Enhanced T cell proliferation as well as Th1 and Th2 cytokine production of CD4+ T cells stimulated with autologous ATI TNM-pulsed mature DC. Immature DC were pulsed with ATI or ATI TNM [15 $\mathrm{g} / \mathrm{mL}]$ and matured with pro-inflammatory cytokines as described in Materials and Methods. After $48 \mathrm{~h}$, mature DC were washed and co-cultivated with autologous CD4 ${ }^{+} \mathrm{T}$ cells for 5 days. (A) T cell proliferation was measured by [ $\left.{ }^{3} \mathrm{H}\right]$-thymidine incorporation, and proliferation index was calculated related to untreated cells. (B-F) Supernatants $(50 \mu \mathrm{L})$ were collected before thymidine administration to determine the production of IL-5, IL-6, IL-10, IL-13, and IFN-gamma by multiplex assay. Results are presented as means \pm SEM from 10 independent experiments/donors. In some cases, cytokine concentrations were below the detection limit so that $n<10$ (IL-5 $n=8$, IL-13 $n=5$, IFN-gamma $n=9)$, ${ }^{\star} P<0.05$, ${ }^{\star \star} P<0.01$.

which seems to correlate with the higher nitration degrees as determined by HPLC-DAD and ELISA. Pre-incubation of the cells with the TLR4 antagonist TAK242 inhibited ATIinduced TLR4 signaling, providing further evidence that ATI stimulate TLR4 directly, as reported in previous studies $(21,22)$. The complete abolishment of TLR 4 activity by TAK242 furthermore allows the conclusion that nitrated ATI also impact the TLR4 agonistic properties directly. As nitration always induces the formation of nitrotyrosine $(1,5,9,27-31)$, it may be hypothesized that nitrotyrosine embedded into the ATI secondary structure itself serves as an amplifier. This hypothesis is supported by our results, showing the lack of enhanced TLR4 stimulation in HeLa TLR4 dual reporter cells upon treatment with unmodified ATI combined with pure nitrotyrosine (Figure S6).

Moreover, nitration of proteins is known to change their structure and function (29). Further investigations would be necessary to clarify to which degree nitration of ATI alters their 
interaction with TLR4 (22), which may explain the observed enhanced TLR4 agonistic activity.

One essential step in TLR4 signaling is the nuclear translocation of the transcription factor NF- $\mathrm{KB}$, controlling the expression of many genes relevant in innate and adaptive immunity $(23,24,32)$. Therefore, we performed several studies on primary immune cells from healthy human donors in vitro. Using fluorescence microscopy, we visualized and quantified nuclear translocation of NF- $\kappa B$ in primary human macrophages after the addition of ATI or nitrated ATI. Under the conditions chosen, native and nitrated ATI provoked comparable nuclear translocation of NF- $\mathrm{kB}$.

Previous studies showed that, not only NF-kB, but also the interferon regulatory factor (IRF-3) gets activated upon ATI treatment (21). Therefore, future investigations should also investigate whether nitrated ATI effect the IRF-3 pathway.

Moreover, we found that the NF- $\mathrm{kB}$ controlled cytokines IL-1 $\beta$, IL-6, IL- 8 , TNF-alpha, and the DC maturation markers CD80 and CD83 (32) were strongly enhanced after treatment of immature DC with ATI and nitrated ATI, which is consistent with previous studies $(21,26)$. This finding indicates that ATI in general induce the maturation of DC, which is an essential step in the induction of $\mathrm{T}$ cell mediated immunity, both via Th1 and Th2 T helper cells, as occurs in autoimmunity and allergy, underpinning the intrinsic immune adjuvant function of (nutritional) ATI $(20,25,26)$.

To investigate the modulation of adaptive immunity by nitrated ATI, we analyzed syngeneic DC-T cell co-cultures. Here, only TNM ATI treated DC induced significantly enhanced $\mathrm{T}$ cell proliferation. Moreover, treatment of DC with ATI or nitrated ATI caused a significantly higher release of Th2 relevant cytokines IL-5, IL-6, IL-10, and IL-13, and of the Th1 cytokine IFN-gamma. For unmodified ATI, this finding is in line with our prior studies on nutritional and inhalative allergies $(25,26)$. Remarkably, nitrated ATI significantly enhanced the production of both Th1 and Th2 cytokines compared to unmodified ATI.

Therefore, it can be concluded that nitrated ATI possess an overall enhanced immune stimulatory potential, including Th1 mediated diseases, as was shown by us before for unmodified ATI in murine inflammatory bowel disease (20). These results make it highly probable that nitrated ATI may more strongly promote inhalative wheat allergies (baker's asthma), NCWS, other allergies, and autoimmune diseases.

Similar findings were reported for other nitrated allergens in the context of allergic airway inflammation or anaphylaxis, but the exact underlying molecular mechanisms are still elusive (1-6). Allergen oligomerization as a side product during the nitration process (e.g., cross-linking of protein-bound tyrosine to form a dimer, or the formation of disulfide bridges) was observed for several proteins, and is discussed as one possible mechanism $(4,30,31,33)$. In this respect, it was suggested that one structural aspect of allergens is their capability to form dimers or oligomers, thereby enhancing their allergenicity (34). Ackaert et al. (4) reported two-fold higher stability for nitrated, as compared to unmodified, Bet $\mathrm{v} 1$, making the allergen more resistant to proteolytic degradation, thus permitting enhanced and prolonged presentation on DC, followed by an enhanced T cell response $(3,4)$. Interestingly, higher molecular weight aggregates instead of oligomers of Bet v 1 (4), and the hypoallergenic Bet v 1d (35) were reported to favor Th1 immunogenicity. Indeed, for all nitration methods used, we found fractions of covalently linked ATI oligomers after the reaction.

Taken together, our results demonstrate that ATI are potent stimulators of innate and adaptive immune responses. In contrast to unmodified ATI, the nitrated ATI exhibit stronger immune stimulatory effects. The observed enhanced immunogenicity of ATI provides a causative chain between stronger TLR4 agonistic effects, leading to enhanced Th1 and Th2 cell activation upon nitration. Future investigations are needed to test how far the degrees of nitration obtained in our study match endogenous ATI nitration under inflammatory conditions, or exogenous nitration, which may occur under certain conditions of wheat growing and processing, e.g., via intensive fertilization, environmental pollution, or nitration during food processing.

\section{DATA AVAILABILITY STATEMENT}

All datasets analyzed for this study are included in the manuscript and the supplementary files.

\section{AUTHOR CONTRIBUTIONS}

DS, UP, KR-S, IB, and KL designed the experiments. KZ, JN, FL, and IB performed the experiments. KZ, JN, FL, JF-N, DS, UP, $\mathrm{IB}$, and $\mathrm{KL}$ analyzed and interpreted the data. CC, JS, FL, KR-S, and IB contributed materials, methods, analysis tools. KZ, IB, and $\mathrm{KL}$ wrote the paper. All authors were involved in the editing and proofreading of the manuscript.

\section{FUNDING}

This work was supported by the Max Planck Graduate Center with the Johannes Gutenberg University Mainz (MPGC). DS was supported for ATI-related work by grants from the German Research Foundation (DFG) (DFG-Schu 646/17-1, DFG-Schu646/20-1, the Collaborative Research Center TR128 Multiple Sclerosis project A08), and the Leibniz Foundation (project Wheatscan). IB was supported by DFG grant BE 4504/3-1.

\section{ACKNOWLEDGMENTS}

The authors acknowledge helpful discussions with the members of the Mainz Program for Chemical Allergology (MPCA) and support from the Max Planck Graduate Center with the Johannes Gutenberg University Mainz (MPGC). KZ and JN acknowledge the support by the IMB Microscopy and Histology Core Facility, as well as the DFG for funding the Opera Phenix High Content Spinning Disk Microscope (Project 402386039).

\section{SUPPLEMENTARY MATERIAL}

The Supplementary Material for this article can be found online at: https://www.frontiersin.org/articles/10.3389/fimmu. 2018.03174/full\#supplementary-material 


\section{REFERENCES}

1. Franze T, Weller MG, Niessner R, Poschl U. Protein nitration by polluted air. Environ Sci Technol. (2005) 39:1673-8. doi: 10.1021/es0488737

2. Gruijthuijsen YK, Grieshuber I, Stocklinger A, Tischler U, Fehrenbach T, Weller MG, et al. Nitration enhances the allergenic potential of proteins. Int Arch Allergy Immunol. (2006) 141:265-75. doi: 10.1159/000095296

3. Karle AC, Oostingh GJ, Mutschlechner S, Ferreira F, Lackner P, Bohle B, et al. Nitration of the pollen allergen bet $\mathrm{v} 1.0101$ enhances the presentation of bet v 1-derived peptides by HLA-DR on human dendritic cells. PLoS ONE (2012) 7:e31483. doi: 10.1371/journal.pone.0031483

4. Ackaert C, Kofler S, Horejs-Hoeck J, Zulehner N, Asam C, Von Grafenstein $\mathrm{S}$, et al. The impact of nitration on the structure and immunogenicity of the major birch pollen allergen Bet v 1.0101. PLoS ONE (2014) 9:e104520. doi: 10.1371/journal.pone.0104520

5. Reinmuth-Selzle K, Ackaert C, Kampf CJ, Samonig M, Shiraiwa M, Kofler $\mathrm{S}$, et al. Nitration of the birch pollen allergen Bet v 1.0101: efficiency and site-selectivity of liquid and gaseous nitrating agents. J Proteome Res. (2014) 13:1570-7. doi: 10.1021/pr401078h

6. Reinmuth-Selzle K, Kampf CJ, Lucas K, Lang-Yona N, Frohlich-Nowoisky J, Shiraiwa $M$, et al. Air pollution and climate change effects on allergies in the anthropocene: abundance, interaction, and modification of allergens and adjuvants. Environ Sci Technol. (2017) 51:4119-41. doi: 10.1021/acs.est.6b04908

7. Galinanes M, Matata BM. Protein nitration is predominantly mediated by a peroxynitrite-dependent pathway in cultured human leucocytes. Biochem J. (2002) 367:467-73. doi: 10.1042/bj20020825

8. Salvemini D, Doyle TM, Cuzzocrea S. Superoxide, peroxynitrite and oxidative/nitrative stress in inflammation. Biochem Soc Transl. (2006) 34:96570. doi: 10.1042/BST0340965

9. Ferrer-Sueta G, Campolo N, Trujillo M, Bartesaghi S, Carballal S, Romero N, et al. Biochemistry of peroxynitrite and protein tyrosine nitration. Chem Rev. (2018) 118:1338-408. doi: 10.1021/acs.chemrev.7b00568

10. Fasano A, Sapone A, Zevallos V, Schuppan D. Nonceliac gluten sensitivity. Gastroenterology (2015) 148:1195-204. doi: 10.1053/j.gastro.2014.12.049

11. Schuppan D, Zevallos V. Wheat amylase trypsin inhibitors as nutritional activators of innate immunity. Dig Dis. (2015) 33:260-3. doi: 10.1159/000371476

12. Zundler S, Neurath MF. Pathogenic $\mathrm{T}$ cell subsets in allergic and chronic inflammatory bowel disorders. Immunol Rev. (2017) 278:263-76. doi: 10.1111/imr.12544

13. Schuppan D, Pickert G, Shfaq-Khan M, Zevallos V. Non-celiac wheat sensitivity: differential diagnosis, triggers and implications. Best Pract Res Clin Gastroenterol. (2015) 29:469-76. doi: 10.1016/j.bpg.2015.04.002

14. Leccioli V, Oliveri M, Romeo M, Berretta M, Rossi P. A New proposal for the pathogenic mechanism of non-coeliac/non-allergic gluten/wheat sensitivity: piecing together the puzzle of recent scientific evidence. Nutrients 9:E1203. doi: 10.3390/nu9111203

15. Walusiak J, Wiszniewska M, Krawczyk-Adamus P, Palczynski C. Occupational allergy to wheat flour. Nasal response to specific inhalative challenge in asthma and rhinitis vs. isolated rhinitis: a comparative study. Int J Occup Med Environ Health (2004) 17:433-40.

16. Tatham AS, Shewry PR. Allergens to wheat and related cereals. Clin Exp Allergy (2008) 38:1712-26. doi: 10.1111/j.1365-2222.2008.03101.x

17. Dupont FM, Vensel WH, Tanaka CK, Hurkman WJ, Altenbach SB. Deciphering the complexities of the wheat flour proteome using quantitative two-dimensional electrophoresis, three proteases and tandem mass spectrometry. Proteome Sci. (2011) 9:10. doi: 10.1186/1477-5956-9-10

18. Finnie C, Melchior S, Roepstorff P, Svensson B. Proteome analysis of grain filling and seed maturation in barley. Plant Physiol. (2002) 129:1308-19. doi: $10.1104 /$ pp.003681

19. Payan F. Structural basis for the inhibition of mammalian and insect alphaamylases by plant protein inhibitors. Biochim Biophys Acta (2004) 1696:17180. doi: 10.1016/j.bbapap.2003.10.012

20. Zevallos VF, Raker V, Tenzer S, Jimenez-Calvente C, Ashfaq-Khan M, Russel $\mathrm{N}$, et al. Nutritional wheat amylase-trypsin inhibitors promote intestinal inflammation via activation of myeloid cells. Gastroenterology (2017) 152:1100-13. doi: 10.1053/j.gastro.2016.12.006

21. Junker Y, Zeissig S, Kim SJ, Barisani D, Wieser H, Leffler DA, et al. Wheat amylase trypsin inhibitors drive intestinal inflammation via activation of tolllike receptor 4. J Exp Med. (2012) 209:2395-408. doi: 10.1084/jem.20102660

22. Cuccioloni M, Mozzicafreddo M, Ali I, Bonfili L, Cecarini V, Eleuteri $\mathrm{AM}$, et al. Interaction between wheat alpha-amylase/trypsin bi-functional inhibitor and mammalian digestive enzymes: Kinetic, equilibrium and structural characterization of binding. Food Chem. (2016) 213:571-8. doi: 10.1016/j.foodchem.2016.07.020

23. Kawai T, Akira S. The role of pattern-recognition receptors in innate immunity: update on Toll-like receptors. Nat Immunol. (2010) 11:373-84. doi: $10.1038 /$ ni. 1863

24. Kawasaki T, Kawai T. Toll-like receptor signaling pathways. Front Immunol. (2014) 5:461. doi: 10.3389/fimmu.2014.00461

25. Bellinghausen I, Weigmann B, Zevallos V, Maxeiner J, Reissig S, Waisman A, et al. Wheat amylase-trypsin inhibitors exacerbate intestinal and airway allergic immune responses in humanized mice. J Allergy Clin Immunol. (2018). doi: 10.1016/j.jaci.2018.02.041. [Epub ahead of print].

26. Zevallos VF, Raker VK, Maxeiner J, Scholtes P, Steinbrink K, Schuppan D. Dietary wheat amylase trypsin inhibitors exacerbate murine allergic airway inflammation Eur J Nutr. (2018). doi: 10.1007/s00394-018-1681-6. [Epub ahead of print].

27. Ischiropoulos H. Protein tyrosine nitration-an update. Arch Biochem Biophys. (2009) 484:117-21. doi: 10.1016/j.abb.2008.10.034

28. Walcher W, Franze T, Weller MG, Poschl U, Huber CG. Liquid- and gasphase nitration of bovine serum albumin studied by LC-MS and LC-MS/MS using monolithic columns. J Proteome Res. (2003) 2:534-42. doi: 10.1021/pr03 $4034 \mathrm{~s}$

29. Radi R. Protein tyrosine nitration: biochemical mechanisms and structural basis of functional effects. Acc Chem Res. (2013) 46:550-9. doi: $10.1021 / \operatorname{ar} 300234 \mathrm{c}$

30. Selzle K, Ackaert C, Kampf CJ, Kunert AT, Duschl A, Oostingh GJ, et al. Determination of nitration degrees for the birch pollen allergen Bet v 1. Anal Bioanal Chem. (2013) 405:8945-9. doi: 10.1007/s00216-013-7324-0

31. Liu F, Reinmuth-Selzle K, Lai S, Weller MG, Poschl U, Kampf CJ. Simultaneous determination of nitrated and oligomerized proteins by size exclusion high-performance liquid chromatography coupled to photodiode array detection. J Chromatogr A. (2017) 1495:76-82. doi: 10.1016/j.chroma.2017.03.015

32. Sakai J, Cammarota E, Wright JA, Cicuta P, Gottschalk RA, Li N, et al. Lipopolysaccharide-induced NF-kappaB nuclear translocation is primarily dependent on MyD88, but TNFalpha expression requires TRIF and MyD88. Sci Rep. (2017) 7:1428. doi: 10.1038/s41598-017-01600-y

33. Kampf CJ, Liu F, Reinmuth-Selzle K, Berkemeier T, Meusel H, Shiraiwa $\mathrm{M}$, et al. Protein cross-linking and oligomerization through dityrosine formation upon exposure to ozone. Environ Sci Technol. (2015) 49:10859-66. doi: 10.1021/acs.est.5b02902

34. Scheurer S, Toda M, Vieths S. What makes an allergen? Clin Exp Allergy (2015) 45:1150-61. doi: 10.1111/cea.12571

35. Zaborsky N, Brunner M, Wallner M, Himly M, Karl T, Schwarzenbacher R, et al. Antigen aggregation decides the fate of the allergic immune response. $J$ Immunol. (2010) 184:725-35. doi: 10.4049/jimmunol.0902080

Conflict of Interest Statement: The authors declare that the research was conducted in the absence of any commercial or financial relationships that could be construed as a potential conflict of interest.

Copyright (C) 2019 Ziegler, Neumann, Liu, Fröhlich-Nowoisky, Cremer, Saloga, Reinmuth-Selzle, Pöschl, Schuppan, Bellinghausen and Lucas. This is an open-access article distributed under the terms of the Creative Commons Attribution License (CC $B Y)$. The use, distribution or reproduction in other forums is permitted, provided the original author(s) and the copyright owner(s) are credited and that the original publication in this journal is cited, in accordance with accepted academic practice. No use, distribution or reproduction is permitted which does not comply with these terms. 